\title{
Presencia y contenidos de los blogs en los principales diarios españoles
}

\section{Por Juan-Miguel Sánchez-Vigil, Juan-Carlos Marcos-Recio y María Olivera-Zaldua}

\begin{abstract}
Resumen: Los medios digitales han acertado con un tipo de información que interesa a los lectores, acostumbrados al uso y consumo de la tecnología que facilita las noticias en diferentes soportes: periódicos en internet, información en redes sociales, documentos informativos en vídeo, etc. Se analiza cómo los blogs han creado un hueco entre los lectores de prensa digital y cómo se han ido implementando en los periódicos digitales hasta contar con sección propia. Se presenta un análisis de los blogs de los principales diarios digitales españoles.

Palabras clave: Blogs, Weblogs, Prensa digital, Periódicos digitales, $A B C$, El país, El mundo, La razón, La vanguardia, Público.

Title: Presence and contents of blogs in the leading Spanish digital newspapers

Abstract: The digital media has proven successful with a specif-

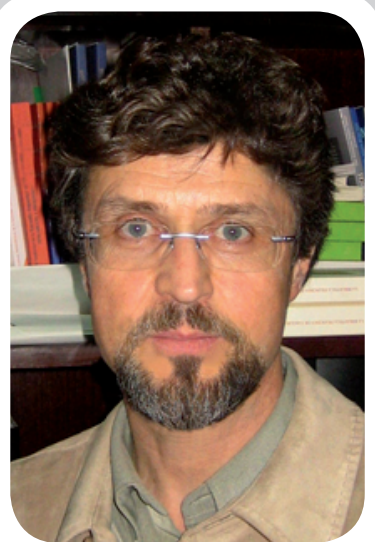

Juan-Miguel Sánchez-Vigil es profesor titular de la Universidad Complutense de Madrid. Especialista en documentación fotográfica y editorial, colabora en varios proyectos de investigación, actualmente en el titulado "Dar clase con la boca cerrada". Ha impartido cursos sobre edición y documentación fotográfica y ha publicado una veintena de libros, entre ellos: La edición en España: industria cultural por excelencia (2009), Revistas ilustradas en España. Del Romanticismo a la Guerra Civil (2008), y El documento fotográfico. Historia, usos y aplicaciones (2006). Codirige el blog Documentación, en Weblogs $m i+d$.
\end{abstract} ic type of information which is

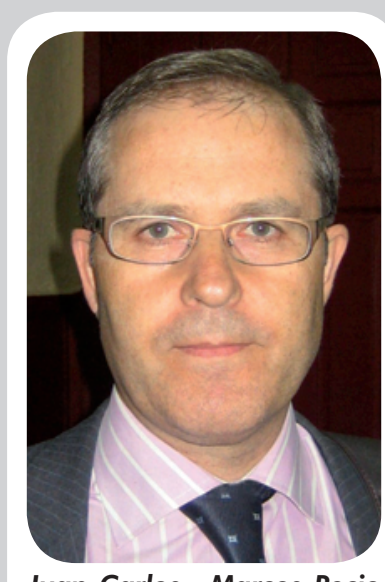

Juan-Carlos Marcos-Recio es profesor titular del Departamento de Biblioteconomía y Documentación de la Universidad Complutense de Madrid. Especialista en documentación en medios y en educación a distancia. Su línea de investigación actual es la documentación y la investigación en la publicidad, donde ha publicado un libro y varios artículos. Es creador del portal Publidocnet (Centro Documental para la Conservación del Patrimonio Publicitario Español), cuyo objetivo es reunir y conservar los estudios, las investigaciones y las campañas publicitarias actuales y antiguas.

http://www.publidocnet.com

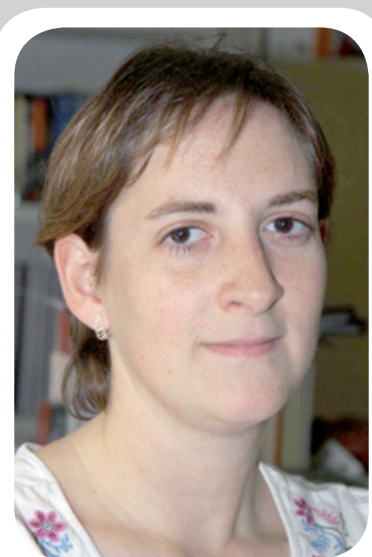

María Olivera-Zaldua es profesora titular interina del Departamento de Biblioteconomía y Documentación de la Universidad Complutense de Madrid (UCM). Documentalista de la editorial Espasa-Calpe. Forma parte del equipo de investigadores del Archivo del Ateneo de Madrid. Ha colaborado en los libros La edición en España, Revistas ilustradas en España y Del daguerrotipo a la Instamatic. Es coautora del libro La música callada de la fotografía. Miradas de Joaquín Turina. Es colaboradora del blog Documentación, en Weblogs $m i+d$.

of great interest to those readers, accustomed to using diverse technology that furnishes them with the news on a variety of platforms: internet newspapers, information on social networks, informative documents on video, etc. This article studies how the blogs have created their own niche among the readers of digital press and how they have been incorporated into the digital newspapers to the point where they now have their own section. A special study is also made of the main Spanish digital daily papers.

Keywords: Blogs, Weblogs, Online journalism, Online newspapers, Digital newspapers, ABC, El país, El mundo, La razón, La vanguardia, Público.

Sánchez-Vigil, Juan-Miguel; Marcos-Recio, Juan-Carlos; Olivera-Zaldua, María. "Presencia y contenidos de los blogs en los principales diarios españoles”. El profesional de la información, 2010, noviembre-diciembre, v. 19, n. 6, pp. 602-609.

DOI: 10.3145/epi.2010.nov.06

\section{Introducción}

Los medios de comunicación son plenamente conscientes de que los blogs interesan a los lectores, acostumbrados ya al uso y consumo de la información en diferentes soportes, y por ello tienen un espacio específico en la prensa digital.
José M. Cerezo (2008, p. 7) indicaba en el prólogo de La blogosfera hispana: "Probablemente lo que pretendemos analizar y reflejar en este libro se parezca muy poco a lo que pueda deparar el futuro en el próximo lustro y muchas de las ilusiones y comentarios aparezcan con el paso del tiempo más como fruto del deseo 
que de la realidad y, como en todo proceso creativo e innovador, fruto de cierta inocencia que se repite en cada nueva revolución tecnológica". Efectivamente, la evolución es tan constante que las conclusiones cambian, pero el estudio puntual es necesario. En la obra citada, Juan Varela (2008, p. 84) ya anunciaba con el título de su colaboración el estado de la cuestión: "Los medios adoptan a los blogs", y calificaba la implantación como la llegada de "un monstruo bíblico", en expresión acertadísima. Desde entonces los blogs han proliferado, y en esa evolución se han producido situaciones que se prestan a nuevos análisis.

El objeto de este artículo es analizar la presencia y contenidos de los blogs en seis de los principales diarios españoles: $A B C, E l$ mundo, $E l$ país, La vanguardia, La razón y Público. Se toma una muestra parcial que se justifica por tratarse de cabeceras de prestigio con alto índice de audiencia. Como punto de partida se ha elaborado un breve marco teórico de presentación para contextualizar la información recopilada.

En la aplicación práctica se han cuantificado los blogs (214 activos en el mes de junio de 2010) y se ha comprobado si disponían de datos de creación, localizando en todos los casos la fecha de la primera entrada para tratar de establecer una cronología. Asimismo se ha considerado la presentación de autores y contenidos, las tipologías sobre materias o categorías específicas, la forma de acceso, la actualización y las posibilidades de recuperación. También se han contabilizado todas las materias y se han asignado nuevas en aquellos casos en que éstas no se indican (caso de La razón). Como trabajo de campo, ha estado abierto a otros temas de interés surgidos durante la experiencia, lo que nos permite conocer la implicación de cada medio.

\section{Presencia de los blogs en la prensa digital}

La presencia de los weblogs en los cibermedios ha sido ya estudiada con carácter general (Blood, 2000; Larrondo, 2005; Noguera-Vivo, 2008) y particular (Carrillo-Durán y Castillo-Díaz, 2009), así como su estructura (Orihuela, 2003; 2004).

Los blogs son sitios de internet dirigidos por una o varias personas en los que se publican posts o entra-

\section{“Los periódicos digitales crean blogs por su necesidad de aproximarse a los lectores"}

das ordenadas cronológicamente, con el más reciente en la parte alta de la página (Blood, 2000; Salaverría, 2005). La mayoría de ellos incluyen información de actualidad y por eso se consideran medios informativos. Jorn Barger fue el primero en usar el término weblog en 1997, que en 1999 Peter Merholz acortó a blog en su bitácora Peterme.com (Millán, 2007).

Noguera-Vivo (2008, p. 26) indica que existen tantas definiciones como autores que las utilizan, señalando que el weblog puede analizarse como una paradoja: "el sufijo log significa anotación o diario personal, y el prefijo web alude a su publicación en la Red, a un acceso universal y abierto, formando una anotación en la Web o como se conoce, un diario personal en red".

La Real Academia incluye la definición en el Diccionario panhispánico de dudas en la acepción bitácora: "Sitio electrónico personal, actualizado con mucha 


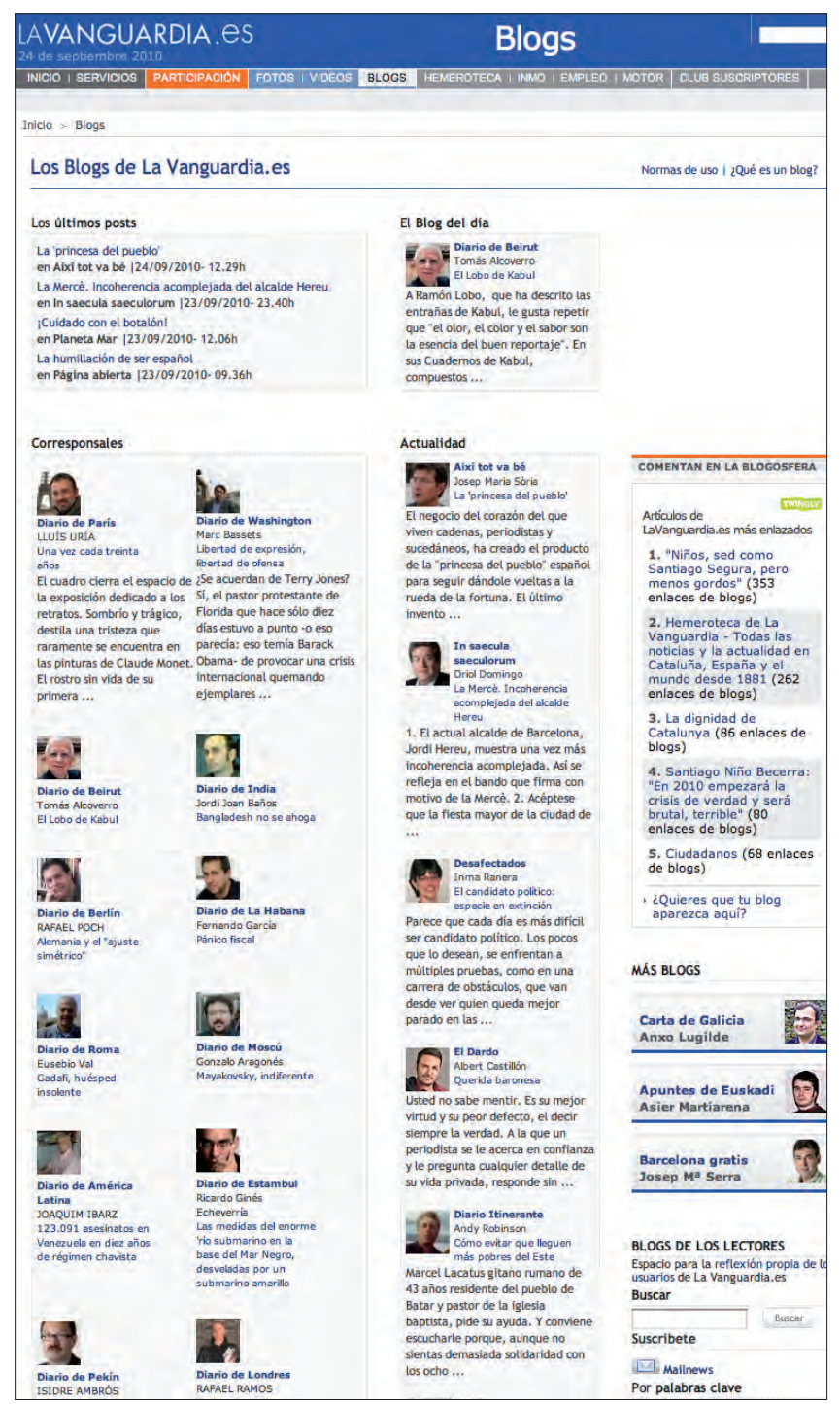

Blogs de La vanguardia, http://www.lavanguardia.es/blogs

frecuencia, donde alguien escribe a modo de diario o sobre temas que despiertan su interés, y donde quedan recopilados asimismo los comentarios que esos textos suscitan en sus lectores" ( $1^{\text {a }}$ ed., octubre 2005).

Dado el carácter de este trabajo, se comprobó si los diarios analizados aportaban definiciones de blog, resultando que sólo lo hacían dos de los seis: El país y La vanguardia.

"Un blog o bitácora es una página en internet, generalmente personal, en la que el usuario puede hacer publicaciones diarias, con un orden cronológico de archivos y la posibilidad de insertar un sistema de comentarios para que los lectores puedan participar" (El país).

"Los blogs son formas de publicación en internet. Son webs que normalmente presentan los contenidos (imagen, texto, audio, vídeo...) de forma cronológica del más al menos reciente, con referencias constantes a otros blogs y webs y que, en la mayoría de los casos, permite la participación de los lectores, que tienen la posibilidad de comentar cada una de las piezas de los autores" (La vanguardia).

La presencia de los blogs en los periódicos digitales obedece en general a su necesidad de aproximarse a los lectores en unos momentos en los que la prensa tradicional viene perdiendo audiencia e ingresos económicos. Según el Libro blanco de la prensa diaria, los diarios en formato digital alcanzaron en 2007 un promedio diario de 6,42 millones de lectores, con un incremento anual del 24\%, datos del Estudio general de medios recogidos en dicho Libro. Los lectores de prensa digital suponían en 2008 el 47,5\% del total, casi diez puntos porcentuales más que en 2007. Esta situación está conformando nuevas formas para los textos informativos y es ahí donde los blogs cobran protagonismo. Ahora bien, la aproximación a los lectores por necesidad conlleva el riesgo de incluir blogs que no respondan a parámetros claros, lo que Carrillo-Durán y Castillo-Díaz (2009, p. 29) han denominado "fenómeno moda". De ahí la importancia de que se expliquen los objetivos y contenidos.

Por otra parte, aunque no sea objeto específico de este trabajo, señalaremos que la apertura de los blogs en los diarios a todo tipo de usuarios está generando confusión, ya que no se advierte con claridad que se trata de dos grupos diferenciados: blogs de profesionales (periodistas y/o expertos en la materia), y de ciudadanos (todos los interesados en participar en el proyecto). Esta situación se constata en las presentaciones de El país y La vanguardia. Mientras el primero explica en la sección La comunidad que "cualquier persona puede crear su propio blog dentro de Elpais.com", el segundo comenta en sus normas de uso: "El nuevo espacio de blogs pretende facilitar la publicación fluida de las reflexiones de los autores habituales para que, gracias a las aportaciones de los usuarios, éstas tengan continuidad y ganen en profundidad". Es decir, que la línea se difumina cuando se mezclan autores habituales con usuarios.

\section{http://lacomunidad.elpais.com}

http://www.lavanguardia.es/blogs/info.html

\section{Estudio de casos en los diarios españoles}

El primer blog informativo en la prensa digital española lo puso en marcha José Cervera con el título Retiario, en El mundo a comienzos de 2004 (RengelLópez, 2005, p. 4), aunque el propio Cervera adelanta la fecha a 2003 en la ficha biográfica de Fesabid 2009. Pionero fue también Ismael Nafría, quien abrió su blog La crónica de internet dedicado a nuevas tecnologías a partir de la sección del mismo título en el digital de $L a$ vanguardia. El primer artículo lo escribió el 11 de enero de 1999 y el último, ya como blog con ese título, el 


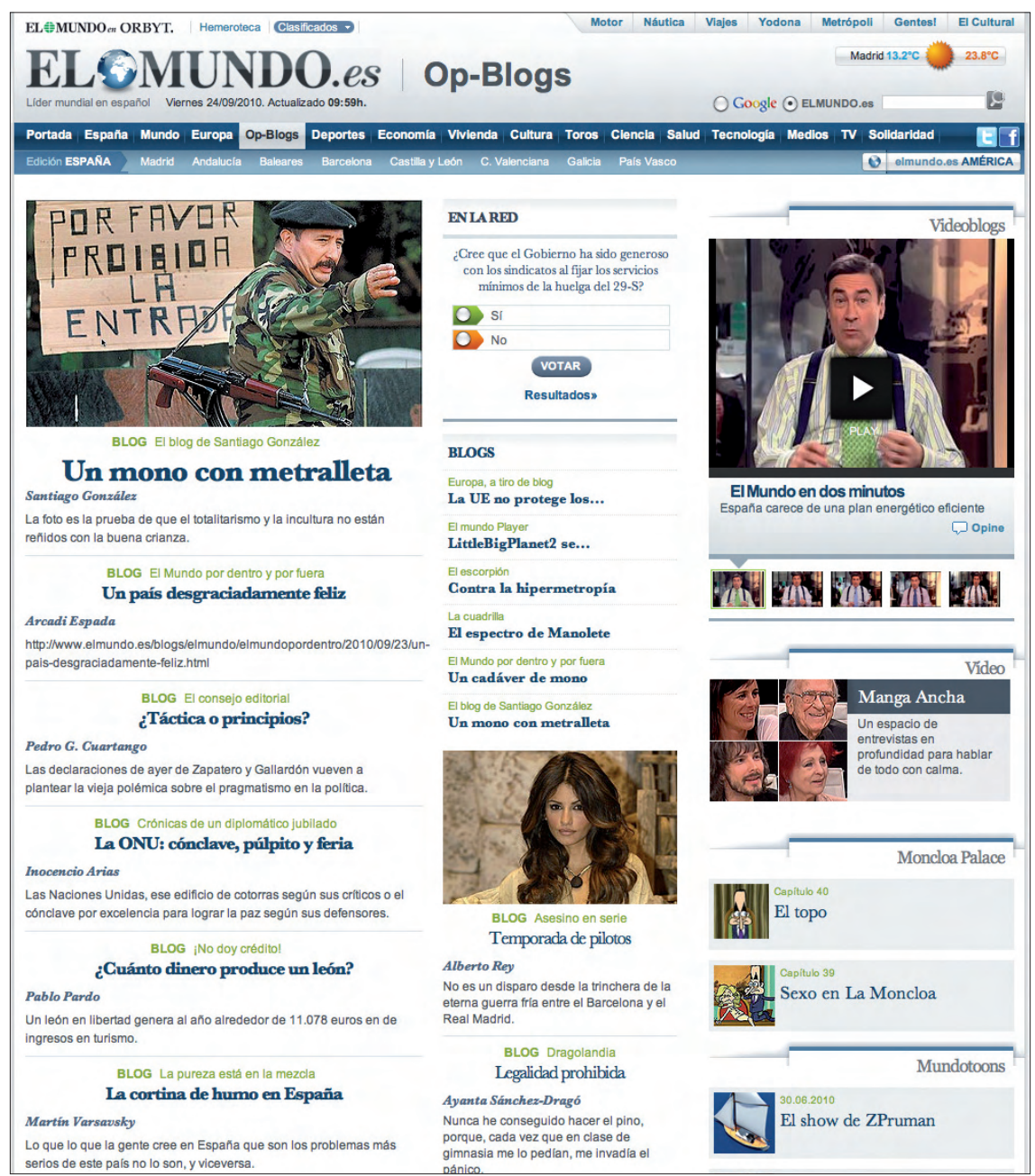

Blogs de El mundo, http://www.elmundo.es/elmundo/blogs.html

de trabajo lo componían Pablo Fernández-Delkader, Fernando Hernández-Puente, Arturo J. Paniagua e Ícaro Moyano-Díaz. Desde entonces el aumento ha sido constante, así como la diversidad de materias, pasando de la tecnología y la ciencia (Retiario, La tejedora y La crónica de internet) a cultura y espectáculos, con muchos ejemplos. El blog Toros, de Rosa JiménezCano, puesto en marcha en mayo de 2007, cuenta con una media de 600 visitas diarias y mantiene sus objetivos de origen: "Comentarios, noticias, vídeos, fotos y crónicas de toros, desde un punto de vista personal, con espacio para la polémica y la subjetividad".

En junio de 2010 los seis diarios seleccionados sumaban un total de 290 blogs, de los que 214 estaban activos y 76 inactivos o cerrados, pero con la información todavía disponible (tabla 1). El mundo destacaba con 49, casi doblando la oferta de La razón, un $30 \%$ más que Público y La vanguardia, y un $20 \%$ por encima de $A B C$ y El país. Es importante el dato de La vanguar-

19 de noviembre de 2008. Aunque está clausurado, sus contenidos aún pueden ser consultados.

http://www.fesabid.org/zaragoza2009/?page_id=1562 http://www.lavanguardia.es/cronica/index.html

$A B C$, El mundo, El país y La razón ofrecen los primeros blogs desde 2005, año que Larrondo (2005) y Guallar (2009) consideran de referencia. Guallar detalla que en el mes de febrero de ese año ya disponían de blogs 20 minutos y El mundo. Público tiene entre sus blogs Las penas del agente Smith, con la primera entrada el 5 de febrero de 2004, migrado por su autor José-María Mateo, ya que el diario salió en 2007. Por su parte, La razón lanzó siete blogs a la vez el 5 de octubre de 2008 sobre diversas materias para equilibrar la oferta con respecto al resto.

El país abrió la sección de blogs en febrero de 2005 con La tejedora: "Un espacio de reunión, una excusa para poner en común los últimos avances en el mundo de la tecnología y aquellas pistas que nos ayudan a todos a navegar con más destreza por la Red. Fue realizada por la sección de información tecnológica de Prisacom (responsable de esta área informativa especialmente en Elpais.es y Cadenaser.com) que está camino de nuevos rumbos profesionales". El equipo dia, con 31 activos y 46 cerrados.

Conocer los objetivos del blog nos parece fundamental. Sin embargo en dos de los diarios ( $A B C$ y $L a$ razón) no se realiza presentación general alguna, como tampoco se hace del autor o responsable en la mayoría. Es esta una cuestión de relevancia para la elección o seguimiento de un blog determinado. No parece importar demasiado este asunto a los profesionales, ya que en la mayoría de los casos no hay posibilidad de contacto.

Por lo que respecta a los contenidos, señalamos la coincidencia de materias con los medios tradicionales, dato que no ha cambiado desde 2004 cuando GarcíaOrosa y Capón-García manifestaron que la agenda temática de los blogs resulta eficaz como segunda estructura de interpretación y jerarquización de la actua-

\begin{tabular}{|l|c|c|}
\hline \multicolumn{1}{|c|}{ Diario } & Activos & Cerrados \\
\hline ABC & 40 & 16 \\
\hline EI mundo & 49 & 4 \\
\hline El país & 39 & 8 \\
\hline Público & 30 & 1 \\
\hline La razón & 25 & 1 \\
\hline La vanguardia & 31 & 46 \\
\hline Total & $\mathbf{2 1 4}$ & $\mathbf{7 6}$ \\
\hline
\end{tabular}

Tabla 1. Blogs activos y cerrados 
lidad. Todos los diarios analizados asignan una materia concreta, y en ciertos casos más de una. Cada uno de ellos establece materias diferentes, por lo que se ha realizado un análisis de las mismas para tratar de establecer un criterio común que permita conocer los contenidos fundamentales.

Las materias convencionales son comunes a todos: Cultura, Deporte, Economía, Sociedad, Comunicación y Tecnología. El diario que estructura sus contenidos en menos materias es La vanguardia, con 6 grandes bloques: Actualidad, Corresponsales, Cultura, Deporte, Sociedad y Tecnología, seguido de Público con 8 apartados: Actualidad, Ciencia, Cultura, Deporte, Economía, Gastronomía, Internacional, Comunicación. El mundo es el que muestra más diversidad, con 13 divisiones (tabla 2).

Dentro del apartado que se ha denominado Especial se incluyen los blogs cuyos contenidos salen de lo convencional con respecto al resto, bien porque son minoritarios o únicos, bien por tratarse de un contenido puntual. Son cinco casos en tres diarios:

- Salud (El mundo)

- Fenómenos paranormales (El mundo)

\begin{tabular}{|l|r|r|r|r|r|r|r|}
\hline \multicolumn{1}{|c|}{ Materia } & ABC & $\begin{array}{c}\text { EI } \\
\text { mundo }\end{array}$ & $\begin{array}{c}\text { EI } \\
\text { país }\end{array}$ & Público & $\begin{array}{c}\text { La } \\
\text { razón }\end{array}$ & $\begin{array}{c}\text { La } \\
\text { vanguardia }\end{array}$ & Total \\
\hline Actualidad & 16 & & & 9 & & 33 & 58 \\
\hline Ciencia & & 5 & & 1 & & & 6 \\
\hline Comunicación & 5 & 4 & 3 & 2 & 2 & & 16 \\
\hline Corresponsales (1) & 6 & 14 & & & & 19 & 39 \\
\hline Cultura & 8 & 6 & 9 & 3 & 6 & 10 & 42 \\
\hline Deporte & 6 & 1 & 8 & 2 & 2 & 8 & 27 \\
\hline Economía & & 1 & 4 & 1 & & & 6 \\
\hline Especial (2) & & 3 & 4 & & 2 & & 9 \\
\hline Gastronomía & 1 & 1 & & 1 & & & 3 \\
\hline Internacional & & 1 & 4 & 1 & 1 & & 7 \\
\hline Nacional / España & & & 3 & & 4 & & 7 \\
\hline Opinión (3) & & 6 & & 11 & 2 & & 19 \\
\hline Sociedad & 5 & 5 & 6 & & 3 & & 22 \\
\hline Tecnología & 4 & 5 & 4 & & 3 & 4 & 20 \\
\hline Viajes & 1 & & 2 & & 1 & & 4 \\
\hline Videoblog & 4 & 1 & & & & & 5 \\
\hline Total & $\mathbf{5 6}$ & $\mathbf{5 3}$ & $\mathbf{4 7}$ & $\mathbf{3 1}$ & $\mathbf{2 6}$ & $\mathbf{7 7}$ & $\mathbf{2 9 0}$ \\
\hline
\end{tabular}

(1) Corresponsales en puntos clave o lugares concretos. (2) Temas específicos relacionados con sucesos puntuales. (3) Contenidos varios o calificados genéricamente como "opinión".

Tabla 2. Materias de los blogs (activos y cerrados)

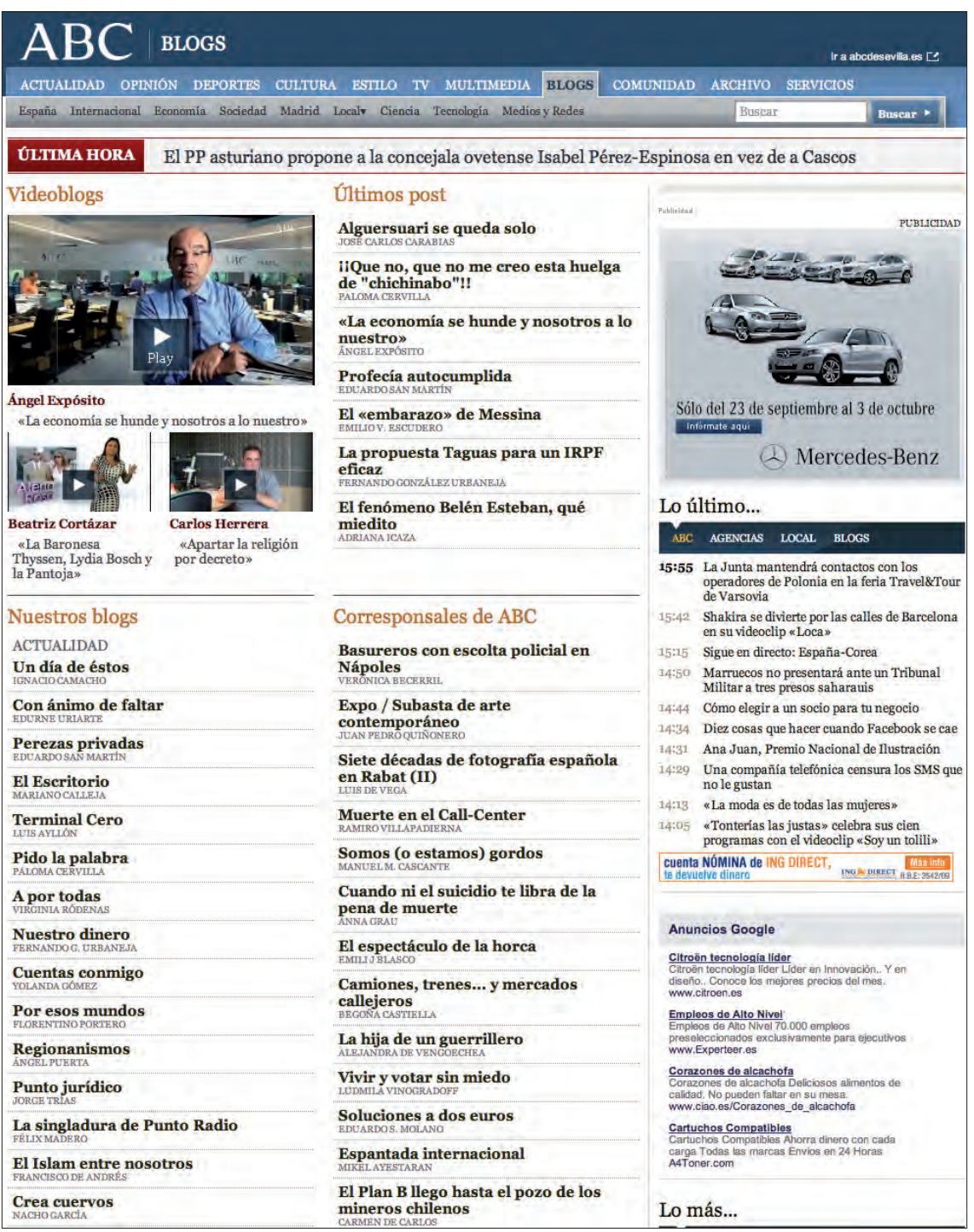

Blogs de $A B C$, http://www.abc.es/blogs

- Sexo (El mundo)

- Medio ambiente (El país)

- Terremoto en Chile (La razón)

El único nombre de sección que resulta algo ilógico es el de Opinión que usa El mundo, pues todos los blogs son básicamente de opinión. A partir de estos datos observamos que temas tan actuales como Medicina, Salud, Medio ambiente o Sexo apenas se tratan, y que otros se relacionan específicamente con sucesos puntuales, como en el caso de las catástrofes (terremotos, inundaciones, etc.). Las materias generales contienen diversos temas y la más significativa es Cultura, con hasta 12 subdivisiones como se observa en la tabla 3. 


\begin{tabular}{|l|c|c|c|c|c|}
\hline \multicolumn{1}{|c|}{ ABC } & El mundo & El país & La razón & Público & La vanguardia \\
\hline 1. Arte & & & & & \\
\hline 2. Cine & Cine & Cine & Cine & Cine & Cine \\
\hline 3. Historia & & & & & \\
\hline 4. Música & Música & Música & & Música & Música \\
\hline 5. Toros & Toros & Toros & Toros & & \\
\hline & 6. Relatos & & & & \\
\hline & & 7. Arquitectura & & & \\
\hline & & 8. Flamenco & & & \\
\hline & & 9. Literatura & & & Libros \\
\hline & & 10. Moda & & & \\
\hline & & & 11. Comics & & \\
\hline & & & 12. Danza & & \\
\hline
\end{tabular}

Tabla 3. Temas de la materia Cultura en los diarios

\section{Características específicas por diarios}

$A B C$

Contiene videoblogs. Se destacan las últimas entradas y las palabras clave en casi todos. No se presenta ni al autor ni los contenidos, salvo excepciones. Se pueden realizar búsquedas parciales por fechas, países y temas.

\section{El mundo}

Contiene videoblogs. Permite la

A partir del análisis se observó que casi todos los blogs contaban con Corresponsales y que es habitual consulta de los que no se actualizan, ofrece resúmenes de los contenidos, se destacan los posts más comentados el uso de Vídeos, resultando dos aspectos más de interés que añadimos a la Presentación y Materia para su comparación. Ninguno de los diarios los tenían todos y La razón no tenía ninguno (tabla 4).

Otra medición que se ha llevado a cabo en el estudio ha sido el número de entradas. Se tomó como referencia el mes de junio de 2010 para conocer tanto la actividad global como la particular. El resultado superó las 1.500 entradas en los 214 blogs, con una media de 7,5 diarias. Esta referencia debe ser matizada ya que, como se observa en la tabla 5 , el $36 \%$ corresponden a $E l$ país, el $29 \%$ a El mundo y el $15 \%$ a $A B C$; es decir que entre tres diarios suman más del $80 \%$ del total. Obviamente es un apunte sobre la actividad general de los blogs en los seis diarios seleccionados, que debe completarse con otros parámetros, en especial la materia sobre la que se informa y el período en el que se lleva a cabo.

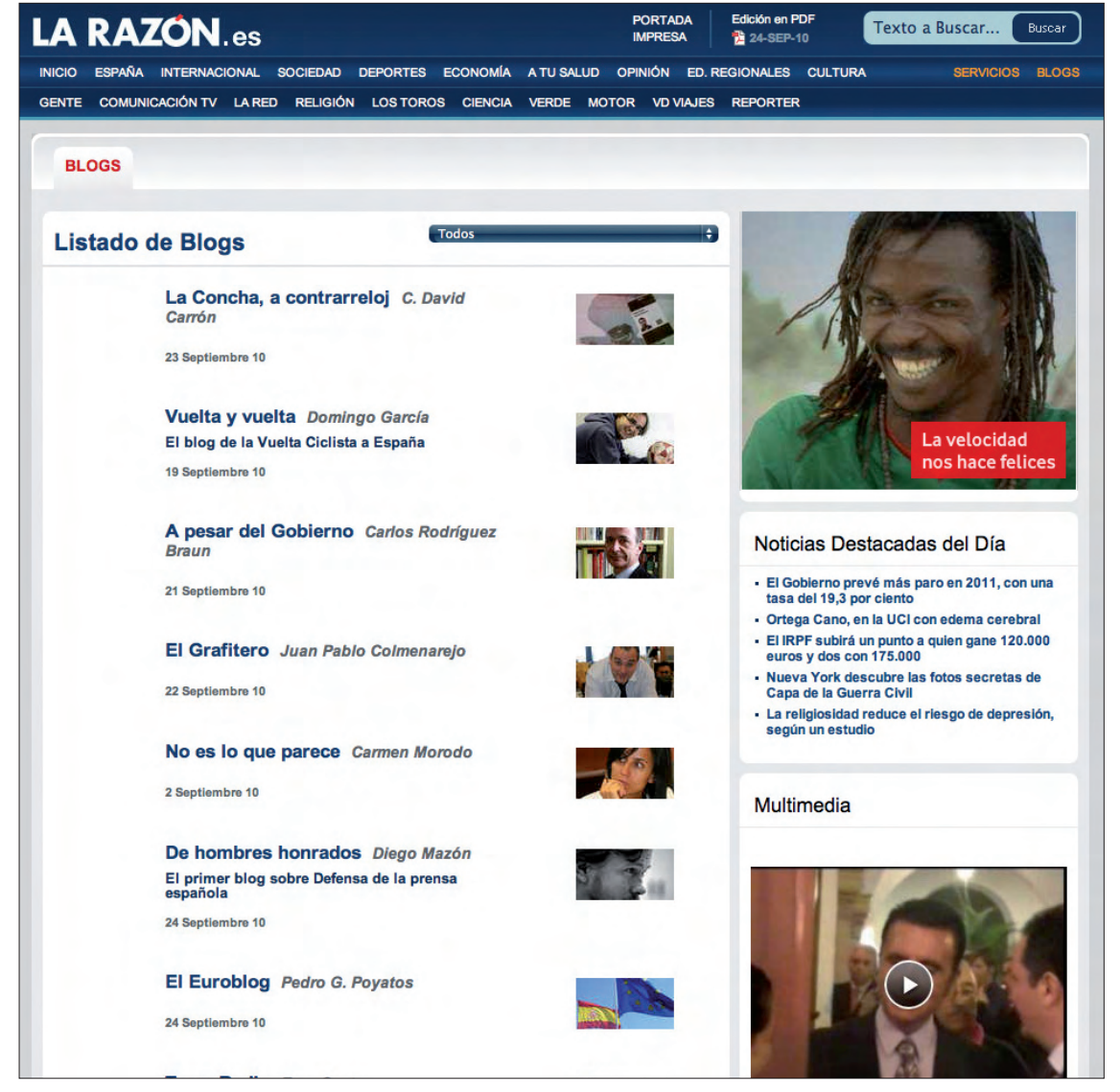

Blogs de La razón, http://www.larazon.es/blogs

\section{"Todos los diarios analizados asignan una o varias materias a los contenidos"}

\begin{tabular}{|l|l|l|l|l|l|l|}
\hline & ABC & El mundo & El país & Público & La razón & La vanguardia \\
\hline Presentación & & & & & & \\
\hline Indicación de materia & & & & & & \\
\hline Videoblog & & & & & & \\
\hline Corresponsales & & & & & & \\
\hline
\end{tabular}

Tabla 4. Comparativa de presentación, materia, videoblogs y corresponsales y establece categorías temporales con entradas antiguas a las que se accede desde la pestaña Ver versiones anteriores (antes de 2010). Los contenidos inclasificables los denomina Otros blogs y es el único diario que contiene la materia Salud.

\section{El país}

Engloba varios temas en una sola categoría y cada blog tiene sólo una materia. Destaca los dos últi- 
mos creados; se presentan todos los contenidos y autores; algunos suplementos del diario disponen de blog propio (EP3 y Babelia); se destacan las categorías o tags en cada blog; en la mayoría se permite la búsqueda por fecha y engloba a los fotoblogs como blogs pero sin destacarlo.

\section{Público}

No dispone de entrada como sección, sino que incluye los blogs en la sección Opinión. No se dan datos de la fecha de entrada de la información sino tan sólo el año; el 25\% (sobre 31) no tiene presentación ni del autor ni de contenidos y en el $75 \%$ restante predominan los datos del autor sobre el contenido. Cada blog tiene asignada una sola materia y la mayoría contiene categorías o tags.

\section{La razón}

La entrada tiene un apartado específico separado de las secciones. No se indica la materia de los contenidos, ni las actualizaciones y no se presentan ni los autores ni los contenidos.

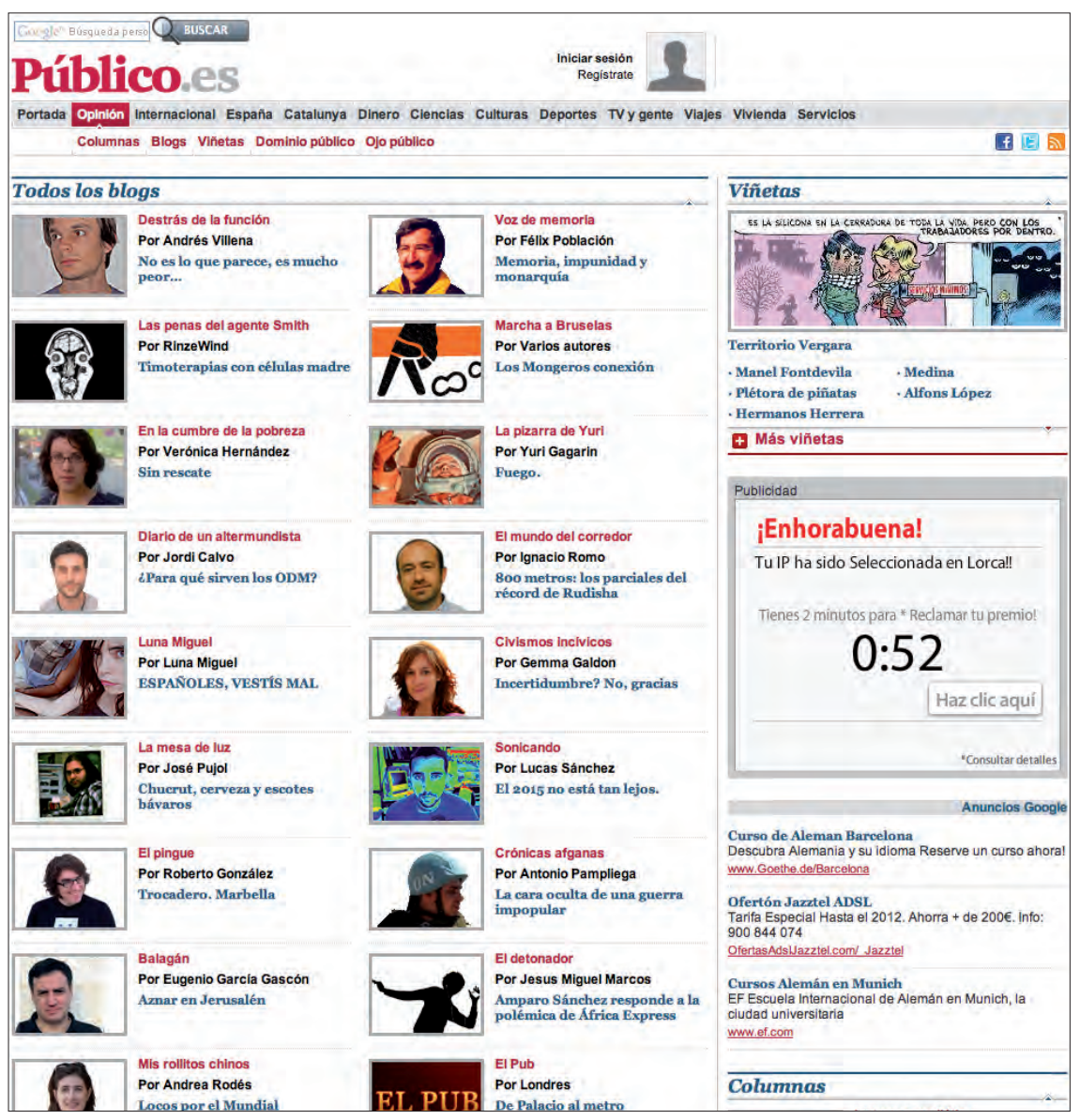

Blogs de Público, http://blogs.publico.es

\section{La vanguardia}

Destacan los blogs de Actualidad y Corresponsales. Presenta un "blog del día" por el interés de su contenido indicando título, autor y parte de la última entrada. Se indican en cada blog los últimos comentarios y permite la búsqueda avanzada.

\section{Conclusiones}

a) Los diarios españoles incorporaron los blogs como sección específica e independiente a partir del año 2005, con el antecedente de José Cervera en el diario El mundo desde 2004.

b) Como norma general no se presentan los ob-

\begin{tabular}{|l|r|r|r|}
\hline \multicolumn{1}{|c|}{ Diario } & Activos & Entradas & \multicolumn{1}{c|}{ \% } \\
\hline ABC & 40 & 236 & 15,1 \\
\hline EI mundo & 49 & 459 & 29,4 \\
\hline El país & 39 & 565 & 36,2 \\
\hline Público (1) & 30 & 96 & 6,2 \\
\hline La razón (2) & 25 & 60 & 3,9 \\
\hline La vanguardia & 31 & 144 & 9,2 \\
\hline Total & $\mathbf{2 1 4}$ & $\mathbf{1 . 5 6 0}$ & $\mathbf{1 0 0 , 0}$ \\
\hline
\end{tabular}

(1) En 11 blogs de Público no se indican las entradas y por tanto en esta tabla no se contabilizan. Si se les aplicara la media del resto de blogs de Público (5 entradas por blog) se obtendrían 152 entradas. (2) Las entradas en La razón son mínimas, con una media de 2,5 entradas al mes en cada blog.

Tabla 5. Número de entradas mensuales en blogs (junio de 2010)

jetivos ni tampoco los autores y/o los contenidos, lo que dificulta la atribución de materia o categoría ya que muchos de los títulos no están relacionados con sus contenidos. Son excepción La vanguardia y El país, que definen el término e incluyen normas de uso.

c) El acceso es directo, con pestaña propia entre las secciones. Es excepción Público, que abre desde la sección Opinión. Todos los sistemas de búsqueda son sencillos, tan sólo La Vanguardia permite una búsqueda avanzada.

d) La vanguardia es el diario que presta mayor atención a los blogs, destacando uno al día en función del interés de su contenido. Es además el único que ofrece los contenidos de todos los blogs cerrados.

e) Se constata que las categorías o materias responden a las convencionales, con cinco temas generales: Actualidad, Cultura, Deporte, Sociedad y Tecnología. Se observa que otras temáticas de gran vigencia, relacionadas con la Salud, Medicina, Ecología o Medio Ambiente no cuentan con blogs.

f) Se considera que los diarios deberían elaborar listados de categorías o materias, a modo de tesauro, con el fin de relacionar los contenidos, de forma que se mantenga la diversidad y sea posible la recuperación de la información. 


\section{Bibliografía citada}

Asociación para la Investigación de Medios de Comunicación. Estudio General de Medios, febr.-nov. 2000.

Asociación para la Investigación de Medios de Comunicación. Estudio general de medios, abr. 2009-mar. 2010.

http://www.aimc.es/aimc.php?izq=egm.swf\&pag_html=si\&op=cuatro\&dc $h=02 \mathrm{egm} / 24 . \mathrm{html}$

Blanco, Adriana. "El quinto poder se teje en la blogosfera". Expansión, 11 oct. 2007.

http://www.expansion.com/2007/10/11/entorno/1044928.html

Blood, Rebecca. "Weblogs: a history and perspective". Rebecca's pocket Sept. 2000.

http://www.rebeccablood.net/essays/weblog_history.html

Carrillo-Durán, María-Victoria; Castillo-Díaz, Ana. "Los blogs en la prensa regional online. La 'actualidad' y la 'actualización' en un nuevo soporte”. Global media journal, 2009, v. 6, pp. 20-36.

http://redalyc.uaemex.mx/src/inicio/ArtPdfRed.jsp?iCve=68712864002

Cerezo, José M. (dir.). La blogosfera hispana: pioneros de la cultura digital. Madrid: Fundación France Telecom, 2008.

http://www.fundacionorange.es/areas/25_publicaciones/la_blogosfera_ hispana.pdf

García-Orosa, Berta; Capón-García, José L. "Las bitácoras o weblogs y la lógica del campo informativo. Un análisis comparativo con la agenda mediática tradicional". Estudios sobre el mensaje periodístico, 2004, n. 10, pp. 113-128.

http://revistas.ucm.es/inf/11341629/articulos/ESMP0404110113A.PDF

Guallar, Javier. "Blogs en publicaciones periodísticas y científicas en España: dos situaciones divergentes. El caso del blog de EPI". Anuario ThinkEPI, 2009, v. 3, pp. 75-87.

http://eprints.rclis.org/17224/

IESE Business School. Libro blanco de la prensa diaria 2009: los editores españoles solicitan ayudas similares a las europeas, 2008.

http://www.iese.edu/aplicaciones/news/view.asp?id=1708\&lang=es

Larrondo, Ainara. "Presencia del formato weblog en los cibermedios: una aproximación a sus usos y funciones". Revista latina de comunicación social, 2005, v. 8 , n. 60

Marcos-Recio, Juan-Carlos. "Una década de periódicos en internet: estrategias documentales". Scire. Representación y organización del conocimiento, 2005 , v. 11 , n. 2 , pp. 63-77.
Marcos-Recio, Juan-Carlos; Sánchez-Vigil, Juan-Miguel; Villegas-Tovar, Ricardo. "Los blogs como soporte de información en bibliotecas y centros de documentación: ¿Qué esperan los usuarios?”. En: VII Congreso de Anabad, 2008, pp. 501-516.

Millán, José-Antonio. "Blog". En: Vocabulario de ordenadores e internet, 2007.

http://jamillan.com/v_blog.htm

Noguera-Vivo, José-Manuel. Blogs y medios: las claves de una relación de interés mиtuo. Madrid: Libros en Red, 2008.

Orihuela, José-Luis. "En medio de los medios: weblogs y periodismo". En: Jornadas presente y futuro del periodismo digital. Facultad de Ciencias Sociales y de la Comunicación del País Vasco (Leoia), 17-18 diciembre 2003

Orihuela, José-Luis. "Los weblogs: de la revolución a la consolidación". Chasqui, Revista latinoamericana de comunicación, n. 85, 2004. http://chasqui.comunica.org/85/orihuela85.htm

Rengel-López, Irene. "La utilización periodística y la integración del formato blog en diarios digitales europeos tradicionales. Los casos del Elmundo.es y Guardian unlimited". Athenea digital, 8, 2005.

Salaverría, Ramón. Redacción periodística en internet. Pamplona: Eunsa, 2005 .

Varela, Juan. "Los medios adoptan a los blogs". En: Cerezo, José M. (dir.) La blogosfera hispana: pioneros de la cultura digital. Madrid: Fundación France Telecom, 2008, pp. 82-106.

Varela, Juan. "Periodismo 3.0, la socialización de la información". Revista Telos, 2005, n. 65, $2^{\text {a }}$ época.

http://periodistas21.blogspot.com/2005/07/periodismo-30-la-socializacinde-la.html

Juan-Miguel Sánchez-Vigil, Juan-Carlos MarcosRecio y María Olivera-Zaldua. Departamento de Biblioteconomía y Documentación de la Universidad Complutense de Madrid. jmsvigil@telefonica.net jmarcos@ccinf.ucm.es molizal@hotmail.com

\section{EPI $n^{\circ} 1$ en el ranking In-Recs \\ $Y$ el Anuario ThinkEPI se estrena con la $7^{\mathrm{a}}$ posición}

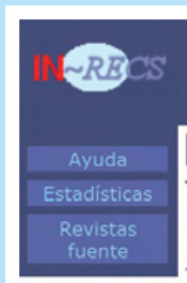

\section{更} 西

Población de revistas: 26

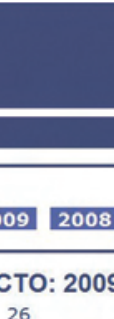

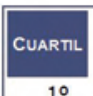

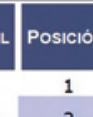

tere

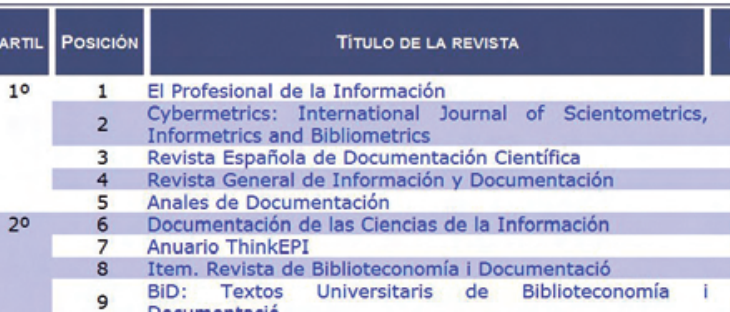

Ordenación por : Indice impacto
INDICE DE IMPACTO BIBLIOTECONOMÍA

\begin{tabular}{l|r} 
Revistas & Articulos
\end{tabular}

Impacto por años

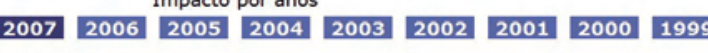

\begin{tabular}{|c|c|}
\hline $\begin{array}{c}\text { INDICE } \\
\text { IMPACTO } \\
2009\end{array}$ & A \\
1.183 \\
0.705 \\
0.607 \\
0.153 \\
0.137 \\
0.113 \\
0.108 \\
0.094 \\
0.092
\end{tabular}

\begin{tabular}{c|c}
$\begin{array}{c}\text { TOTAL } \\
\text { ARTICULOS }\end{array}$ & $\begin{array}{c}\text { TOTA } \\
\text { CITAS }\end{array}$ \\
\hline 71 & 84 \\
17 & 12 \\
51 & 31 \\
65 & 10 \\
51 & 7 \\
44 & 5 \\
92 & 10 \\
53 & 5 \\
65 & 6
\end{tabular}

Según el Índice de Revistas Españolas de Ciencias Sociales (In-Recs), elaborado por el Grupo EC3 (Evaluación de la Ciencia y de la Comunicación Científica) de la Universidad de Granada: http://ec3.ugr.es/in-recs/ii/ Biblioteconomia-fecha-2009. htm la revista El profesional de la información ha conseguido situarse en primer lugar, de entre 26 revistas españolas de biblioteconomía y documentación. 